\title{
Investigations on the unsteady behaviour of cavitation and the corresponding pressure field
}

\author{
par R. Boehm, B. Stoffel, G. Ludwig
}

Darmstadt University of Technology, Germany

Laboratory for Turbomachinery and Fluid Power

\section{IINTRODUCTION}

The occurence of leading edge cavitation, for instance in rotodynamic pumps and water turbines, still requests extensive experimental and theoretical investigations. Although a certain amount of cavitation is tolerated during operation of turbomachinery in many cases, cavitation is mostly linked with a loss of efficiency, noise generation and cavitation erosion. Characteristics of the cavitation, which seem to be mainly responsible for the erosive aggressivity, are the type of cavitation, volume of vapour, unsteady behaviour of cavitation and unsteady pressure field. The characteristics of the non-cavitating flow field, e.g. pressure distribution, type of boundary layer and flow separation can also have an influence on the cavitation behaviour for comparable Reynolds numbers.

Our investigations, which are still in progress and are financially supported by the German Community of Research (DFG), aim at finding the dependence of the erosive aggressivity on the typical cavitation characteristics and the correlation between cavitating and non-cavitating flow field, e.g. areas of separated flow, type of boundary layers and pressure distribution. Furthermore, the investigations shall give information on the characteristics of the 3-dimensional behaviour of cavitation and on the influence of secondary flows on the erosive aggressivity.

The knowledge of these influences on the cavitation aggressivity will help to estimate the risk of erosion in an early stage of design, for instance on the basis of the CFDcalculated pressure distribution.

Investigations concernant le comportement instationnaire

de la cavitation et le champ de pression correspondant

Les investigations systématiques concernant le flux de cavitation et non-cavitation ont été réalisées. L'enregistrement simultané des images avec des temps d'exposition courts et des pressions dynamiques permet l'interprétation des caractéristiques dynamiques macroscopiques de la cavitation dans diverses conditions de cavitation.

L'évaluation à l'aide d'un système de traitement d'images conduit à des contours moyens de cavité (liés au volume de vapeur), une fourchette de fluctuations et à des idées sur l'influence de l'apparition dynamique de la cavitation sur le champ de pression instationnaire.

Des mesures continues de pression dans le domaine temporel fournissent des informations sur le champ de pression instationnaire et les fréquences perdues. Par mesures et calculs sur les flux non cavitants, on peut prouver (dans la limite des méthodes) qu'aucune séparation de flux n'est nécessaire pour l'apparition de cavitation laminaire. 


\section{II —TEST SECTION AND MEASURE- MENT EQUIPMENT}

\subsection{Test Section}

The experimental investigations are performed in a 2D test section, which is part of a closed test rig. The test section contour contains a flat part with a forward facing step as the cavitating profile (fig. 1). The profile is designed as being rotatable to change the angle of incidence (and thereby the pressure distribution on the wall. The profile is equipped with 7 pressure tappings which can be used for static measurements. Two transparent windows, on the top and in the sidewall, give the opportunity for flow visualisation with the aid of laser light sheet and CCD-camera and application of other measuring techniques, e.g. Laser Doppler Velocimety (LDV).

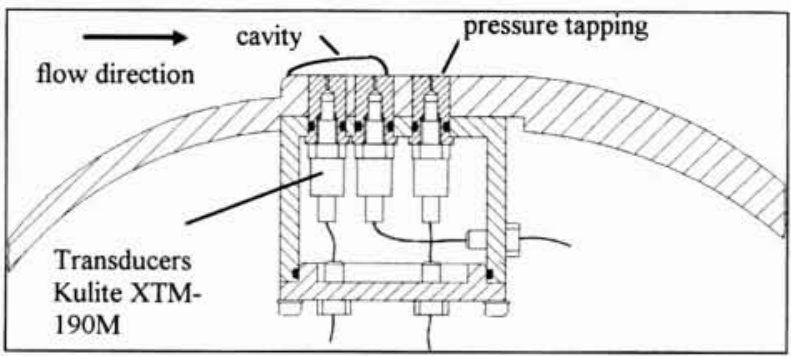

1. Drawing of profile with forward facing step and arrangement of dynamic pressure transducer.

\subsection{Dynamic Pressure Measurement}

Furthermore, the cavitating profile is equipped with dynamic pressure tappings and piezoresistive pressure transducers, Kulite XTM-190M, located in the closure region of the cavitation. The cavities in front of the transducer membranes are filled with silicon oil as a transmitter fluid to detect the unsteady pressure resulting from cavitation fluctuations. Thus, the unintended measurement of microjet pressure signals and attenuation or phase shift resulting from free gas bubbles in these cavities are avoided to the greatest possible extent. A pressure tapping of $0.5 \mathrm{~mm}$ diameter represents the connection of the oil filled cavity and the surface of the profile (fig. 1).

\subsection{Laser Light Sheet and Image Processing}

An argon ion laser (fig. 2) is used for flow visualisation with laser light sheet (LLS) technique and for LDV measurements, respectively. For LLS-measurements, the beam was aligned into an acousto-optical modulator (AOM, Bragg cell) for short pulsation of laser beam intensity. The rise time of the pulses depends on the wave length, beam diameter and the sound velocity inside the Bragg crystal material (e.g. $\mathrm{TeO}_{2}$ ). A typical rise time for the AOM used was $350 \mathrm{~ns}$. The AOM is controlled by a TTL trigger signal, representing the intended pulse length. This signal is provided by a signal generator, getting an input burst signal from the image processing board. The burst signal marks an image which is grabbed by the frame grabber. Since the AOM control signal is also used as a trigger for the signal memory recorder the assignment of exposure and pressure signal is possible. After passing the AOM the beam is transmitted using a glass fiber and finally spread to a light sheet by a cylinder lens.

The illuminated contours of the cavities are detected with a CCD-camera and an image processing system. The evaluation with a search routine leads to the coordinates of the cavity coun-

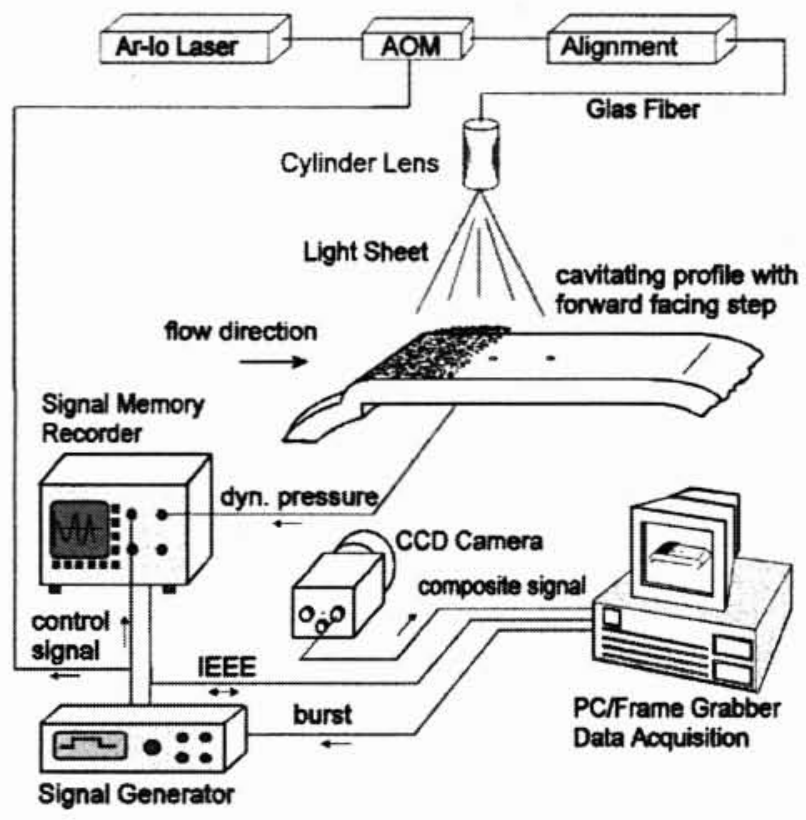

2. Presentation of light sheet and image processing measurement equipment.

tour for each image. Therefore, shedding ranges, lengths frequency histograms and cavity volumes can be calculated for any number of images of one cavitating flow condition.

\section{III $\square$ CAVITATION AND PRESSURE FLUC- TUATION}

Typical (inverted) images of a fixed cavity cycle (not taken in chronological order) with an exposure time of $20 \mu \mathrm{s}$ are presented in figure 3 where the flow direction is from left to right. The contours of the cavities are determined using the search routine (in inverted images : thin black line). The first image shows clearly the contour of the closed cavity in the formation and growth phase. The contour at the end of the cavity close to the wall demonstrates the existence of a reentrant flow. The instantaneous pressure at the exposure time window is plotted at the transducer positions. Since the first sensor $(p 1)$ is covered by the closed cavitation pocket, the measured pressure of 105 mbar is close to the vapour pressure of $42 \mathrm{mbar}$ (water temperature $30^{\circ} \mathrm{C}$ ). An unexpectedly high fraction of liquid phase in a leading edge cavity was found by Stutz [4] by the means of optical sensors. Assuming that the pocket contains vapour as well as water, the transducer detects an average pressure of these two phases. Although piezoresistive transducers and amplifiers for dynamic measurements are in use, the transducers in combination with the pressure holes are not able to detect those high frequency pressure fluctuations of passing single bubbles alternating with layers of water (moreover, the detection of these fluctuations was not intended). Thus, the measured mean pressure has to be a function of the fraction of vapour and water inside the cavity. The distance from the leading edge and the influence of the reentrant flow can be observed for the second transducer $(p 2=231 \mathrm{mbar})$. The flow approaching the wall in the wake of the cavity results in a pressure of $2,026 \mathrm{bar}$, which is above the magnitude of approximately 1.8 bar for the non-cavitating flow.

The 2nd image presents the cavitation just before a total breakoff and the formation of a cloud. Pressure p1 is above and $\mathrm{p} 3$ below the magnitude which is found during the growth of the cavity. The breakoff (3rd image) is linked with low pressures in the newly growing cavity and the cloud. 


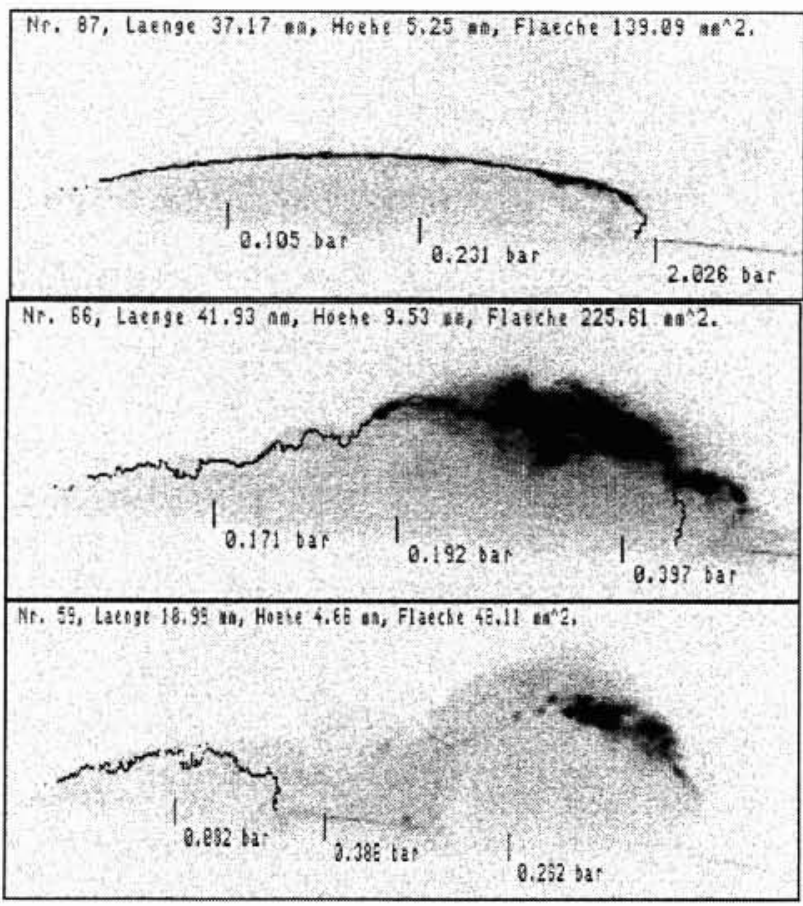

3. Typical fixed cavity cycle.

Figure 4 presents average cavitation contours for 3 velocities and 3 different mean cavity lengths. Each contour (line) is a superposition of about 1000 images. The mean cavity lengths $L_{c}=10.5,23$ and $38 \mathrm{~mm}$ were adjusted by visual observation with a stroboscobic light source. The image processing evaluation demonstrates, that this adjustment was chosen too short. This deviation can be explained from the differences of lengths adjustments and evaluations during experiment. The image processing system evaluates only the closed cavity, to the greatest possible extent without cloud. (Only if the closed cavity and the cloud are still linked, a few $\mu$ s after the breakoff, the cloud is included in the evaluation). This is intended, because for statistical evaluation the vapour volume should not be calculated from 'new' and 'old' cavities, as well, in one frame. On the other hand, the adjustment by eye is very probably influenced by the occurence of clouds, interpreted as part of the cavity.

Comparing this effect with earlier investigations [1] according to the aggressivity distribution for a sheet cavity (pressure pulse measurements by variation of transducer position along the cavity), it has been found that the highest and strongest pulses (resulting from microjets) occur at the eye-adjusted cavity end.

Figure 5 shows representative results of these investigations, where $S / L r$ is the dimensionless cavity length and

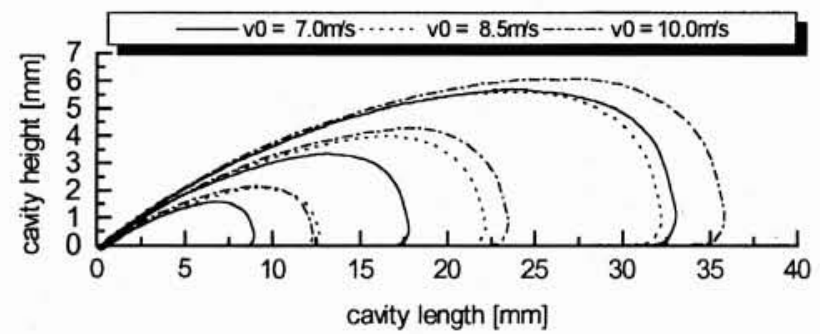

4. Cavitation contours of 9 flow conditions, evaluated by image processsing system.

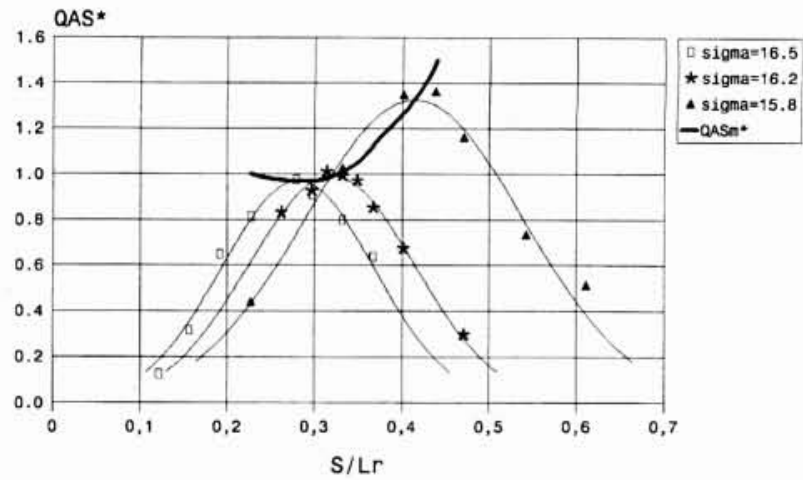

5. Variation of position of pressure pulse transducer for 3 cavity lengths.

QAS* has the meaning of a sum of squared amplitudes of the pressure pulses, which correlates to the intensity of erosive aggressivity. The approximated magnitudes result in a Gaussian distribution with the maximum at the eye-adjusted end of the cavitation. Since we know from the image processing investigations described above, that the evaluation leads to shorter lengths, the strong microjet pulses seem to be mostly a result of cloud cavitation. In future investigations, this shall be verified by quasi-simultaneous acquisition of images and pressure pulses.

According to the determination of the range of the cavity length for single images (in this case 1000 frames, $20 \mu \mathrm{s}$ exposure time), histograms of instantaneous lenghts can be obtained (fig. 6). Significant is the similarity of length histogramms and QAS*-distributions and the large fluctuation range of long in comparison to short cavities.

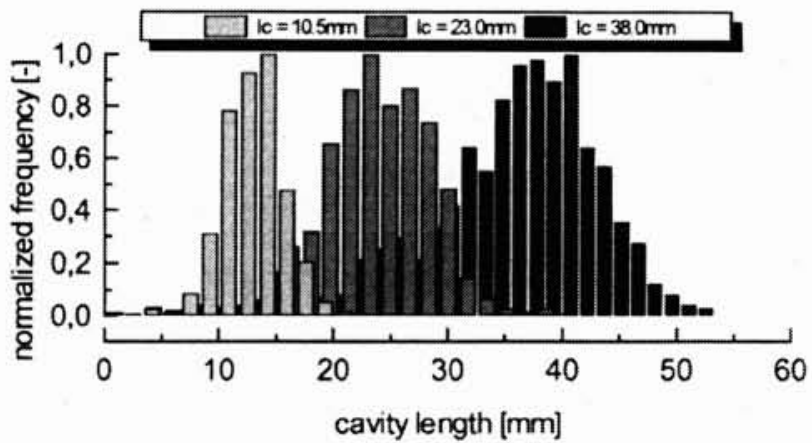

6. Histogram of instantaneous lengths.

Figure 7 demonstrates pressure signals which were recorded simultaneously for a mean cavity length of $L_{c} / L_{\text {ref }}=0.21$ $\left(L_{c}=10.5 \mathrm{~mm}, L_{r e f}=50 \mathrm{~mm}\right)$. For these measurements, the mean end of the cavity is close to the first dynamic pressure transducer $p 1$. Obviously, $p 1$ shows the lowest mean pressure. As a verification of measuring correctly, the mean pressures are comparable with the mean static pressure found in [1].

The peaks in the time domain signal of $p 1$ occur, when the breakoff has finished and the surrounding flow of the cavity is approaching the pressure hole.

The frequency spectrum of $p 1$ shows a peak frequency of about $144 \mathrm{~Hz}$ which seems to be below a magnitude representative of a fluctuation frequency for the given length. Transducers $p 2$ and $p 3$ show a peak frequency of $\cong 590 \mathrm{~Hz}$, which is closer to the expected shedding frequency of a short cavity. The high frequency components in the ampli- 


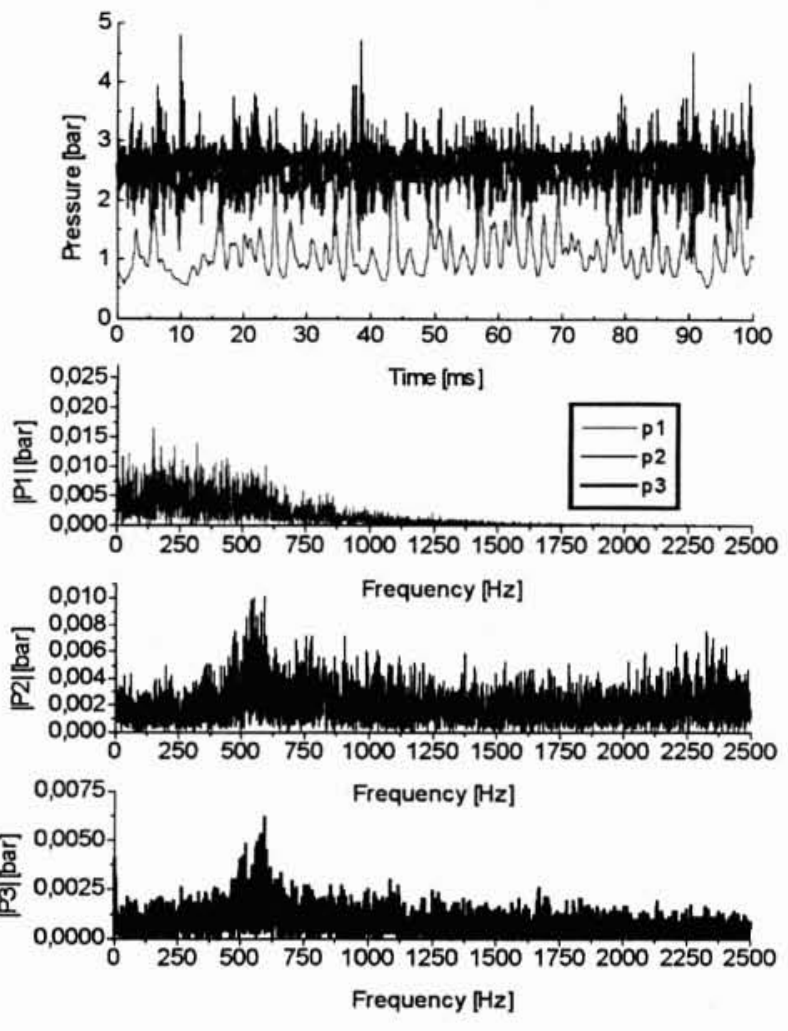

7. Pressure signals in time and frequency domain, $L_{c} / L_{r e f}=0.21$.

tude spectrum of $p 2$ and $p 3$ can not be ignored. Since these sensors are located in the wake of the cavity, clouds are passing the pressure holes. Assuming that these clouds generate high frequency signals $(>2 \mathrm{kHz})$, this effect can be explained. Wang et al. [4] reported on calculations of shock waves generated by collapses of clouds. In these investigations it was found that the far-field acoustic impulses are strongly correlated to the volume and the void fraction of the cloud. The pressure signals in the closure region exceed the maximum pressure (neglecting the influence of microjets) which can be predicted by steady flow calculation (fig. 3, top). The maximum pressure is detected during the growth of the cavity, when the end passes the location of the pressure transducer. Therefore, a stream of water approaches the wall (with a perpendicular velocity component) and it can be calculated as following (considering incompressible liquid and only hydrodynamic effects). $V c$ has the meaning of a characteristic velocity, assuming vapour existing at the interface of the closed cavity and the surrounding liquid :

$$
p_{\max }=\frac{1}{2} \rho V_{c}^{2}+p_{v}
$$

The characteristic velocity can be calculated from (see also [1])

$$
V_{c}=V_{0} \sqrt{1+\sigma}
$$

using Bernoulli's equation (for steady flow), where $V_{0}$ is the reference pressure at a location upstream the profile and $\sigma$ is the dimensionless cavitation number:

$$
\sigma=\frac{p-p_{v}}{\frac{1}{2} \rho V_{0}^{2}}
$$

The characteristic velocities for the cavity lengths $L_{c} / L_{\text {ref }}=$ $0.21,0.46$ and 0.76 (mean length of cavities at the pressure transducers) and the resulting dynamic pressure for the approaching flow is therefore :

$$
\begin{array}{lll}
L_{\mathcal{L}} / L_{r e f}=0.21 & & \\
V_{c}=30.9 \mathrm{~m} / \mathrm{s} & \rightarrow & p_{\max }=4,7 \mathrm{bar} \\
\boldsymbol{L}_{\mathcal{C}} \boldsymbol{L}_{\text {ref }}=\mathbf{0 . 4 6} & & \\
V_{c}=28.9 \mathrm{~m} / \mathrm{s} & \rightarrow & p_{\max }=4,2 \mathrm{bar} \\
\boldsymbol{L}_{\mathcal{C}} \boldsymbol{L}_{\text {ref }}=\mathbf{0 . 7 6} & & \\
V_{c}=27.6 \mathrm{~m} / \mathrm{s} & \rightarrow & p_{\max }=3.8 \mathrm{bar}
\end{array}
$$

Figure 8 presents the pressure for a normalized length of $L_{c} L_{\text {ref }}=0.76$ and exhibits high and short pressure peaks for the transducer which is located in the region of mean cavity length $(p 3)$. These peaks exceed the predicted dynamic pressure of an approaching flow in some cases. This could actually be another indication for a superposition of cloud collapses and unsteady hydrodynamic flow effects, which cannot be predicted with a steady flow calculation. A few of the high peaks are followed by a local minimum and smaller peaks, which possibly could be interpreted as a detachment of smaller vapour structures from the closure region. For the cavity fluctuation frequencies and therefore the Strouhal number $S t=F_{c}{ }^{*} L_{c} / V_{c}$, it results :

$$
\begin{array}{lll}
L_{c} / L_{\text {ref }}=0.21 & & \\
F_{c} \approx 564 \mathrm{~Hz} & \rightarrow & S t \approx 0.23[-] \\
L_{c} / L_{\text {ref }}=0.46 & & \\
F_{c} \approx 244 \mathrm{~Hz} & \rightarrow & S t \approx 0.21[-] \\
L_{c} / L_{\text {ref }}=\mathbf{0 . 7 6} & & \\
F_{c} \approx 144 \mathrm{~Hz} & \rightarrow & S t \approx 0.20[-]
\end{array}
$$
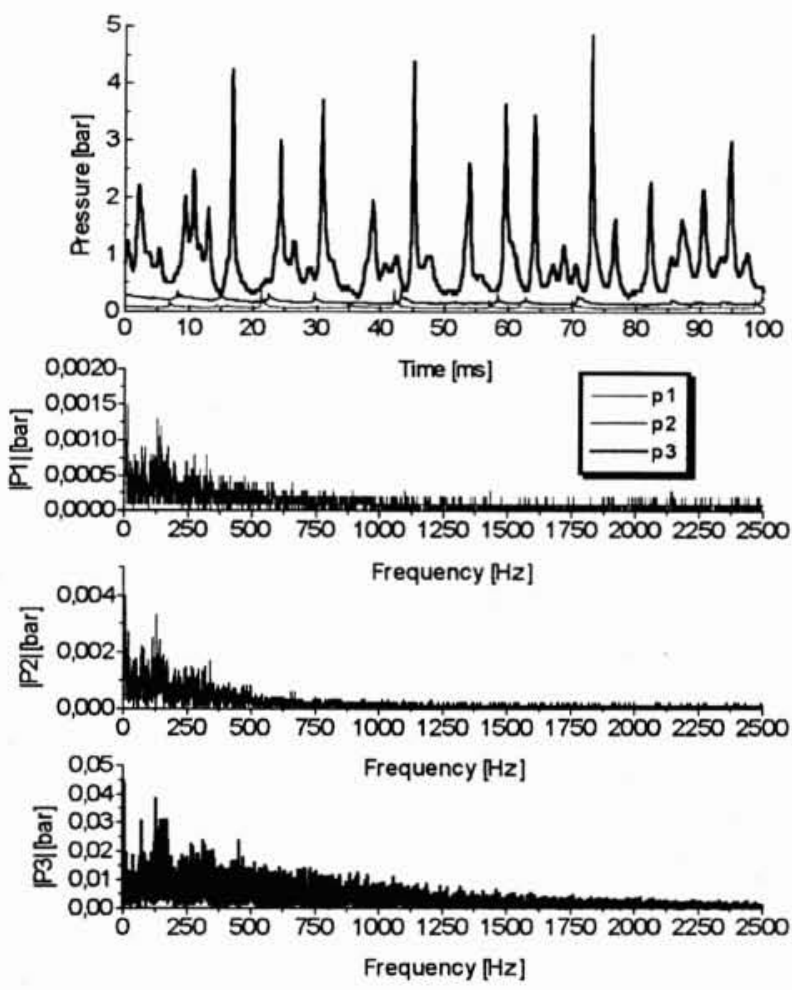

8. Pressure signals in time and frequency domain, $L_{c} / L_{r e f}=0.76$.

\section{IV — NON-CAVITATING FLOW}

As mentioned in the introduction, another aim of the research project is to investigate the influence of characteristics of the non-cavitating flow on the unsteady behaviour 
and the aggressivity of leading edge cavitation. These investigations were carried out using numerical methods (CFDcalculation with the code FIDAP), and experimentally with the aid of Laser Doppler Velocimetry.

A special objective of flow field investigations was to find out whether a separation of the non-cavitating flow exists and possibly influences the sheet cavitation. The flow near the profile was "scanned " with the LDV-system (as described by the pattern in fig. 9) for Reynolds-numbers comparable with the cavitation conditions. Figure 10 shows velocities for various distances from the profile $(z=0.5-2.0$ $\mathrm{mm}$ ) versus the coordinate $x$ along the profile. At a location of $x=2 \mathrm{~mm}$ (leading edge) the highest velocity component $v_{x}$ is occuring in the layer nearest to the profile. For a location of $x=4 \mathrm{~mm}$ this effect inverts and the influence of boundary layer can be observed (smaller velocites nearer to the wall). An indication for negative velocities typically occuring in separated flows was not observed. If there exists a separated flow, it was not detectable, since the measurement distance to the profile of $z=0.5 \mathrm{~mm}$ represents the smallest possible distance (because of reflections disturbing the photo-multiplier). CFD-calculations (see fig. 11, turbulence intensity at inlet set to $5 \%$ ) verified the LDV-measurements and represented non-separated flow in the interesting area, even for the highest angle of incidence $\left(10^{\circ}\right)$ which was chosen for the calculations. Thus, obviously the boundary layers are getting a sufficient amount of energy from the turbulent main flow, which allows to pass zones of high positive pressure gradients without detectable separation bubbles. Though the typical characteristics of cavitation

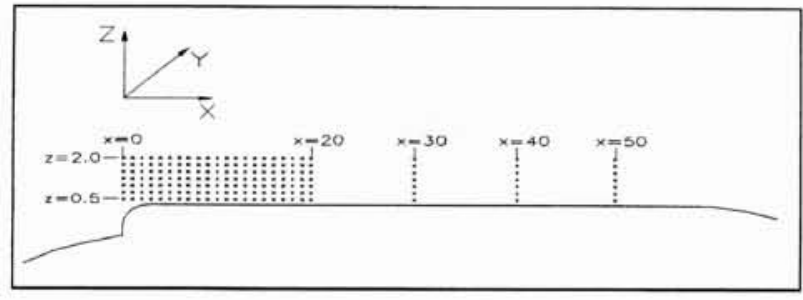

9. Pattern of LDV-measurements.

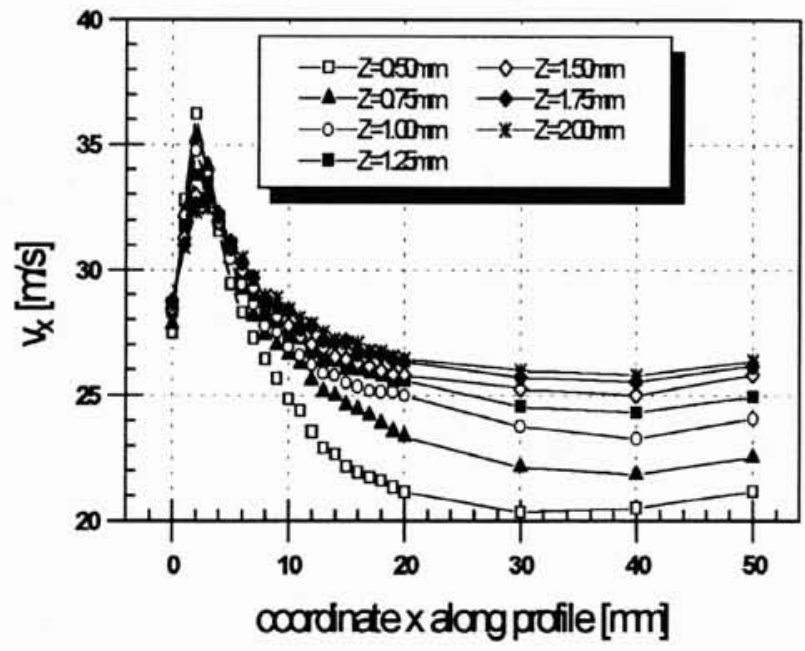

10. LDV-measurements on the forward facing step, angle of incidence $0^{\circ}$.

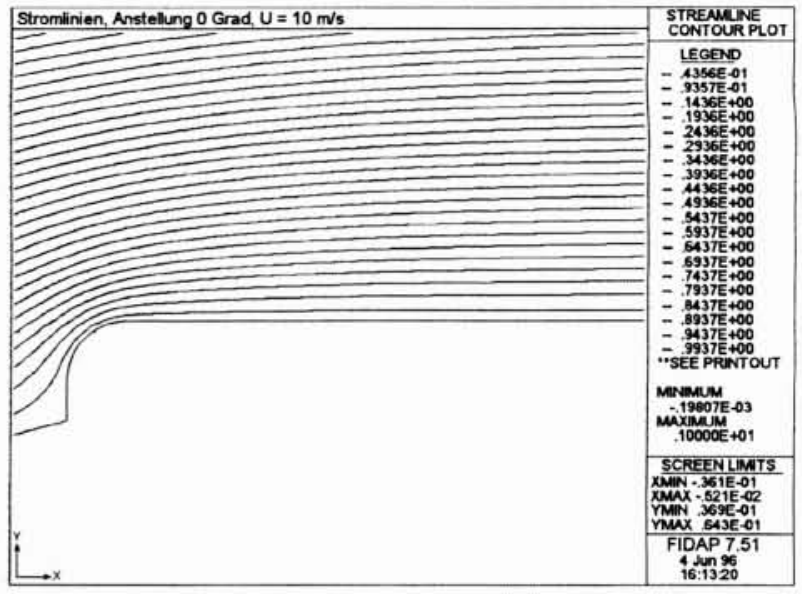

11. Calculated streamlines in the area around the forward facing step.

inception indicated the existence of laminar separation bubbles, these could not be observed with the aid of the LDV-system.

\section{$\mathrm{V} \square \mathrm{CONCLUSIONS}$}

- Measurements of the unsteady pressure in combination with the laser light sheet techniques can provide information on unsteady flow field, and shedding frequencies.

- With the aid of image processing it is possible to obtain fluctuation ranges of cavity length fluctuations and the size of the cavities.

- Determination of mean cavity lengths and comparisons with pressure pulse measurements can show the main source (cloud, cavity) of the erosive aggressivity.

- Further considerations will concern the driving pressure differences representing an influence on erosive aggressivity, correlations with non-cavitating $2 \mathrm{D}$ and $3 \mathrm{D}$ flow and the clarification of the dependence on laminar separation bubbles.

\section{REFERENCES}

[1] SChUller W. (1995). - « Akustische Signale und lokale Druckimpulse als $\mathrm{Ma} B$ für die hydrodynamische Intensität der Kavitation (Acoustic Signals and Local Pressure Pulses as a Measure for the Hydrodynamic Cavitation Intensity) ", Doctor Thesis, Darmstadt University of Technology, Germany

[2] Stutz B. (1996). - «Analyse de la Structure Diphasique et Instationnaire de Poche de Cavitation ", Doctor Thesis, Institut National Polytechniques de Grenoble.

[3] Knapp, Dally, Hammit (1970). - « Cavitation », McGrawHill Book Company.

[4] Wang Y.-C., Brennen C.E. (1995), - « The Noise Generated by the Collapse of a Cloud of Cavitation Bubbles ", 1995 ASME/JSME Fluids Engineering and Laser Anemometry Conference and Exhibition, August 13-18, Hilton Head, South Carolina, FED-Vol. 226. 